\title{
Solution and Stability of Euler-Lagrange-Rassias Quartic Functional Equations in Various Quasinormed Spaces
}

\author{
Heejeong Koh and Dongseung Kang \\ Department of Mathematical Education, Dankook University, 126 Jukjeon, Suji, Yongin, Gyeonggi 448-701, Republic of Korea \\ Correspondence should be addressed to Dongseung Kang; dskang@dankook.ac.kr
}

Received 9 July 2013; Accepted 3 September 2013

Academic Editor: Nicole Brillouet-Belluot

Copyright (C) 2013 H. Koh and D. Kang. This is an open access article distributed under the Creative Commons Attribution License, which permits unrestricted use, distribution, and reproduction in any medium, provided the original work is properly cited.

\begin{abstract}
We obtain the general solution of Euler-Lagrange-Rassias quartic functional equation of the following $f(a x+b y)+f(b x+a y)+$ $(1 / 2) a b(a-b)^{2} f(x-y)=\left(a^{2}-b^{2}\right)^{2}[f(x)+f(y)]+(1 / 2) a b(a+b)^{2} f(x+y)$. We also prove the Hyers-Ulam-Rassias stability in various quasinormed spaces when $b=1$.
\end{abstract}

\section{Introduction}

One of the interesting questions concerning the stability problems of functional equations is as follows: when is it true that a mapping satisfying a functional equation approximately must be close to the solution of the given functional equation? Such an idea was suggested in 1940 by Ulam [1] as follows. Let $G_{1}$ be a group and let $G_{2}$ be a metric group with the metric $d(\cdot, \cdot)$. Given $\varepsilon>0$, does there exist a $\delta>0$ such that if a function $h: G_{1} \rightarrow G_{2}$ satisfies the inequality $d(h(x y), h(x) h(y))<\delta$ for all $x, y \in G_{1}$ then there is a homomorphism $H: G_{1} \rightarrow G_{2}$ with $d(h(x), H(x))<$ $\varepsilon$ for all $x \in G_{1}$ ? In other words, we are looking for situations when the homomorphisms are stable; that is, if a mapping is almost a homomorphism, then there exists a true homomorphism near it. In 1941, Hyers [2] considered the case of approximately additive mappings in Banach spaces and satisfying the well-known weak Hyers inequality controlled by a positive constant. The famous Hyers stability result that appeared in [2] was generalized in the stability involving a sum of powers of norms by Aoki [3]. In 1978, Rassias [4] provided a generalization of Hyers Theorem which allows the Cauchy difference to be unbounded. During the last decades, stability problems of various functional equations have been extensively studied and generalized by a number of authors [5-10]. In particular, Rassias [11] introduced the Euler-Lagrange type quadratic functional equation

$$
f(r x+s y)+f(s x-r y)=\left(r^{2}+s^{2}\right)[f(x)+f(y)],
$$

for fixed reals $r, s$ with $r \neq 0, s \neq 0$. Also, Jun and Kim [12] proved the Hyers-Ulam-Rassias stability of a Euler-Lagrange type cubic mapping as follows:

$$
\begin{aligned}
f(a x+b y)+f(b x+a y)= & (a+b)(a-b)^{2}[f(x)+f(y)] \\
& +a b(a+b) f(x+y),
\end{aligned}
$$

where $a \neq 0, b \neq 0, a \pm b \neq 0$, for all $x, y \in X$. Several EulerLagrange type functional equations have been investigated by numerous mathematicians; c.f. for example, [13-15].

And Rassias [16] investigated stability properties of the following quartic functional equation:

$$
\begin{aligned}
f(x & +2 y)+f(x-2 y)+6 f(x) \\
& =4 f(x+y)+4 f(x-y)+24 f(y) .
\end{aligned}
$$

It is easy to see that $f(x)=x^{4}$ is a solution of (3) by virtue of the identity

$$
\begin{aligned}
& (x+2 y)^{4}+(x-2 y)^{4}+x^{4} \\
& \quad=4(x+y)^{4}+4(x-y)^{4}+24 y^{4} .
\end{aligned}
$$

For this reason, (3) is called a quartic functional equation. Also, Chung and Sahoo [17] determined the general solution of (3) without assuming any regularity conditions on the unknown function. In fact, they proved that the function 
$f: \mathbb{R} \rightarrow \mathbb{R}$ is a solution of (3) if and only if $f(x)=$ $A(x, x, x, x)$, where the function $A: \mathbb{R}^{4} \rightarrow \mathbb{R}$ is symmetric and additive in each variable. Lee and Chung [18] introduced a quartic functional equation as follows:

$$
\begin{aligned}
f(a x+y)+f(a x-y) \\
=a^{2} f(x+y)+a^{2} f(x-y) \\
\quad+2 a^{2}\left(a^{2}-1\right) f(x)-2\left(a^{2}-1\right) f(y),
\end{aligned}
$$

for fixed integer $a$ with $a \neq 0, \pm 1$.

In this paper, we consider the following a generalized quartic functional equation:

$$
\begin{aligned}
f(a x+b y)+f(b x+a y)+\frac{1}{2} a b(a-b)^{2} f(x-y) \\
=\left(a^{2}-b^{2}\right)^{2}[f(x)+f(y)] \\
+\frac{1}{2} a b(a+b)^{2} f(x+y),
\end{aligned}
$$

for fixed integers $a$ and $b$ such that $a \neq 0, b \neq 0, a \pm b \neq 0$, for all $x, y \in X$. In fact, the generalized quartic functional equation (6) is following from the spirit of the pioneering Euler-Lagrange quartic functional equation (3) as well as Euler-Lagrange quadratic functional equation (1) introduced by Rassias: see [16] and [11], respectively. For the same reason as (1), (2), and (3), we call (6) a Euler-Lagrange-Rassias quartic functional equation. First of all, we obtain the general solution of Euler-Lagrange-Rassias quartic functional equation. To prove the stability problem for the Euler-LagrangeRassias quartic functional equation on various quasi-normed spaces, we may consider the following:

$$
\begin{aligned}
f(a x & +y)+f(x+a y)+\frac{1}{2} a(a-1)^{2} f(x-y) \\
& =\left(a^{2}-1\right)^{2}[f(x)+f(y)]+\frac{1}{2} a(a+1)^{2} f(x+y),
\end{aligned}
$$

for fixed integer $a$ with $a \neq 0, a \neq \pm 1$, for all $x, y \in X$.

We will use the following definitions to prove HyersUlam-Rassias stability for the Euler-Lagrange-Rassias quartic functional equation in the quasi- $\beta$-normed and quasi fuzzy $\beta$-normed spaces. Let $\beta$ be a real number with $0<\beta \leq 1$ and let $\mathbb{K}$ be either $\mathbb{R}$ or $\mathbb{C}$.

Definition 1. Let $X$ be a linear space over a field $\mathbb{K}$. A quasi $\beta$-norm $\|\cdot\|$ is a real-valued function on $X$ satisfying the following statements:

(1) $\|x\| \geq 0$ for all $x \in X$ and $\|x\|=0$ if and only if $x=0$,

(2) $\|\lambda x\|=|\lambda|^{\beta} \cdot\|x\|$ for all $\lambda \in \mathbb{K}$ and all $x \in X$,

(3) there is a constant $K \geq 1$ such that $\|x+y\| \leq K(\|x\|+$ $\|y\|)$ for all $x, y \in X$.

The pair $(X,\|\cdot\|)$ is called a quasi- $\beta$-normed space if $\|\cdot\|$ is a quasi- $\beta$-norm on $X$. The smallest possible $K$ is called the modulus of concavity of $\|\cdot\|$. A quasi- $\beta$-Banach space is a complete quasi- $\beta$-normed space.

A quasi $\beta$-norm $\|\cdot\|$ is called a $(\beta, p)$-norm $(0<p \leq 1)$ if (3) takes the form $\|x+y\|^{p} \leq\|x\|^{p}+\|y\|^{p}$ for all $x, y \in X$. In this case, a quasi $\beta$-Banach space is called a $(\beta, p)$-Banach space; see $[19,20]$.

In 1984, Katsaras [21] and Wu and Fang [22] independently introduced a notion of a fuzzy norm and they gave the generalization of the Kolmogoroff normalized theorem for a fuzzy topological linear space. Since then, some mathematicians have defined fuzzy metrics and norms on a linear space from various points of view; see [23-27]. In 2003, Bag and Samanta [23] modified the definition of Cheng and Mordeson [28]. Bag and Samanta [23] introduced the following definition of fuzzy normed spaces. The notion of fuzzy stability of functional equations was given in the paper [29].

Definition 2. Let $X$ be a real vector space. A function $N: X \times$ $\mathbb{R} \rightarrow[0,1]$ is called a fuzzy norm on $X$ if for all $x, y \in X$ and all $s, t \in \mathbb{R}$

$$
\begin{aligned}
& \left(N_{1}\right) N(x, t)=0 \text { for } t \leq 0 ; \\
& \left(N_{2}\right) x=0 \text { if and only if } N(x, t)=1 \text { for all } t>0 ; \\
& \left(N_{3}\right) N(c x, t)=N(x, t /|c|) \text { if } c \neq 0 ; \\
& \left(N_{4}\right) N(x+y, s+t) \geq \min \{N(x, s), N(y, t)\} ; \\
& \left(N_{5}\right) N(x, \cdot) \text { is a nondecreasing function of } \mathbb{R} \text { and } \\
& \quad \lim _{t \rightarrow \infty} N(x, t)=1 \text {; } \\
& \left(N_{6}\right) \text { for } x \neq 0, N(x, \cdot) \text { is continuous on } \mathbb{R} .
\end{aligned}
$$

The pair $(X, N)$ is called a fuzzy normed vector space.

Mirmostafaee [30] introduced a notion for a quasi fuzzy p-normed space as follows.

Definition 3. By a quasi fuzzy norm, one means a real vector space $X$, with a fuzzy subset $N$ of $X \times \mathbb{R}$ and some $K \geq 1$ such that all axioms of fuzzy normed space in Definition 2 except $\left(N_{4}\right)$ and

$$
\begin{array}{r}
\left(N_{4}^{\prime}\right) \\
N(x+y, K(s+t)) \geq \min \{N(x, s), N(y, t)\} \\
(x, y \in X, s, t>0)
\end{array}
$$

hold.

A quasi fuzzy normed space $(X, N)$ which satisfies

$$
\begin{array}{r}
N(x+y, \sqrt[p]{s+t}) \geq \min \{N(x, \sqrt[p]{s}), N(y, \sqrt[p]{t})\} \\
(x, y \in X, s, t>0),
\end{array}
$$

for some $0<p \leq 1$, is called a quasi fuzzy p-norm.

Definition 4. Let $X$ be a real vector space. A quasi fuzzy $p$ norm $N: X \times \mathbb{R} \rightarrow[0,1]$ is called a quasi fuzzy $(\beta, p)$-norm on $X$ if $\left(N_{3}\right)$ in Definition 2 takes the form 
$\left(N_{3}^{\prime}\right)$

$$
N(c x, t)=N\left(x, \frac{t}{|c|^{\beta}}\right) \quad(c \neq 0,0<\beta \leq 1) .
$$

Example 5. Let $(X,\|\cdot\|)$ be a real normed space. Define

$$
N(x, t)= \begin{cases}\frac{t}{t+\|x\|} & \text { when } t>0, t \in \mathbb{R} \\ 0 & \text { when } t \leq 0,\end{cases}
$$

where $x \in X$. Then $(X, N)$ is a quasi fuzzy $(\beta, p)$-normed space.

Note that when $p=1$, we call the quasi fuzzy $(\beta, p)$-norm a quasi fuzzy $\beta$-norm.

Definition 6. Let $(X, N)$ be a quasi fuzzy $\beta$-normed vector space. A sequence $\left\{x_{n}\right\}$ in $X$ is said to be convergent or converge if there exists an $x \in X$ such that $\lim _{n \rightarrow \infty} N\left(x_{n}-\right.$ $x, t)=1$ for all $t>0$. In this case, $x$ is called the limit of the sequence $\left\{x_{n}\right\}$ and one denotes it by $N-\lim _{n \rightarrow \infty} x_{n}=x$.

Definition 7 . Let $(X, N)$ be a quasi fuzzy $\beta$-normed vector space. A sequence $\left\{x_{n}\right\}$ in $X$ is called Cauchy if for each $\varepsilon>0$ and each $t>0$ there exists an $n_{0} \in \mathbb{N}$ such that, for all $n \geq n_{0}$ and all integer $d>0$, one has $N\left(x_{n+d}-x_{n}, t\right)>1-\varepsilon$.

It is well known that every convergent sequence in a quasi fuzzy $\beta$-normed vector space is Cauchy. If each Cauchy sequence is convergent, then the quasi fuzzy $\beta$-normed space is said to be quasi fuzzy complete and the quasi fuzzy $\beta$ normed vector space is called a quasi fuzzy Banach space.

\section{Euler-Lagrange-Rassias Quartic Functional Equations}

Let $X, Y$ be real vector spaces. In this section, we will investigate that the functional equation (3) is equivalent to the presented functional equation (6).

Lemma 8. A mapping $f: X \rightarrow Y$ satisfies the functional equation (3) if and only if $f$ satisfies

$$
\begin{aligned}
f(2 x+y)+f(x+2 y)= & 9 f(x)+9 f(y) \\
& +9 f(x+y)-f(x-y),
\end{aligned}
$$

for all $x, y \in X$.

Proof. It follows from [31, 32].

Theorem 9. A mapping $f: X \rightarrow Y$ satisfies the functional equation (3) if and only if $f$ satisfies the functional equation (7).

Proof. It is easy to verify that $f(0)=0$ by letting $x=y=0$ in (3). We will show this induction on $a$. Lemma 8 implies that it is true when $a=2$, and we may assume it holds for all $a$. Now, letting $x=(a-1) x+y$ and $y=x$ in (3), we have

$$
\begin{aligned}
f((a+1) x+y)+f((a-3) x+y) \\
=4 f(a x+y)+4 f((a-2) x+y) \\
\quad+24 f(x)-6 f((a-1) x+y),
\end{aligned}
$$

for all $x, y \in X$. After the switching $x$ and $y$ in the previous equation (13),

$$
\begin{aligned}
f(x+ & (a+1) y)+f(x+(a-3) y) \\
= & 4 f(x+a y)+4 f(x+(a-2) y) \\
& +24 f(y)-6 f(x+(a-1) y),
\end{aligned}
$$

for all $x, y \in X$. Adding two equations (13) and (14), we have

$$
\begin{aligned}
f(x+ & (a+1) y)+f(x+(a+1) y) \\
= & -[f((a-3) x+y)+f(x+(a-3) y)] \\
& +4[f(a x+y)+f(x+a y)] \\
& +4[f((a-2) x+y)+f(x+(a-2) y)] \\
& -6[f((a-1) x+y)+f(x+(a-1) y)] \\
& +24[f(x)+f(y)],
\end{aligned}
$$

for all $x, y \in X$. The induction steps imply that

$$
\begin{aligned}
f(x+ & (a+1) y)+f(x+(a+1) y) \\
= & -\frac{1}{2} a^{3} f(x-y)-\frac{1}{2} a^{2} f(x-y) \\
& +\frac{1}{2} a^{3} f(x+y)+\frac{5}{2} a^{2} f(x+y) \\
& +4 a f(x+y)+2 f(x+y) \\
& +\left(a^{4}+4 a^{3}+4 a^{2}\right)[f(x)+f(y)] \\
= & -\frac{1}{2}(a+1)((a+1)-1)^{2} f(x-y) \\
& +\frac{1}{2}(a+1)((a+1)+1)^{2} f(x+y) \\
& +\left((a+1)^{2}-1\right)^{2}[f(x)+f(y)],
\end{aligned}
$$

for all $x, y \in X$. Hence we have

$$
\begin{aligned}
f((a+1) x+y)+f(x+(a+1) y) \\
+\frac{1}{2}(a+1)((a+1)-1)^{2} f(x-y) \\
=\left((a+1)^{2}-1\right)^{2}[f(x)+f(y)] \\
\quad+\frac{1}{2}(a+1)((a+1)+1)^{2} f(x+y),
\end{aligned}
$$

for all $x, y \in X$, as desired. 
Note that $f(a x)=a^{4} f(x)$ by letting $y=0$ in (7).

Lemma 10. A mapping $f: X \rightarrow Y$ satisfies the functional equation (7) if and only if $f$ satisfies the functional equation (6).

Proof. It is easy to show that $f(0)=0$ and $f(a x)=a^{4} f(x)$ by putting $x=y=0$ and $y=0$ in (7), respectively. By letting $y=b y$ in (7), we have

$$
\begin{aligned}
f(a x+b y)+f(x+a b y)+\frac{1}{2} a(a-1)^{2} f(x-b y) \\
=\left(a^{2}-1\right)^{2}[f(x)+f(b y)]+\frac{1}{2} a(a+1)^{2} f(x+b y) .
\end{aligned}
$$

Also, switching $x$ and $y$ in the above equation and then adding two equations, we get

$$
\begin{aligned}
f(a x+ & b y)+f(b x+a y) \\
= & -[f(a b x+y)+f(x+a b y)]+\left(a^{2}-1\right)^{2} \\
& \times\left[f(x)+f(y)+b^{4} f(x)+b^{4} f(y)\right] \\
& +\frac{1}{2} a(a+1)^{2}[f(b x+y)+f(x+b y)] \\
& -\frac{1}{2} a(a-1)^{2}[f(b x-y)+f(x-b y)] .
\end{aligned}
$$

Then (7) implies that

$$
\begin{aligned}
f(a x+b y)+f(b x+a y)+\frac{1}{2} a b(a-b)^{2} f(x-y) \\
=\left(a^{2}-b^{2}\right)^{2}[f(x)+f(y)]+\frac{1}{2} a b(a+b)^{2} f(x+y) .
\end{aligned}
$$

Corollary 11. A mapping $f: X \rightarrow Y$ satisfies the functional equation (3) if and only if $f$ satisfies the functional equation (6).

\section{Stability in Quasi- $\beta$-Normed Spaces}

Throughout this section, let $X$ be a quasi- $\beta$-normed space and let $Y$ be a quasi $\beta$-Banach space with a quasi $\beta$-norm $\|\cdot\|_{Y}$. Let $K$ be the modulus of concavity of $\|\cdot\|_{Y}$. We will investigate the Hyers-Ulam-Rassias stability problem for the functional equation (7). For a given mapping $f: X \rightarrow Y$ and all fixed integers $a$ with $a \neq 0, a \neq \pm 1$, let

$$
\begin{aligned}
D_{a} f(x, y):= & f(a x+y)+f(x+a y) \\
& +\frac{1}{2} a(a-1)^{2} f(x-y) \\
& -\left(a^{2}-1\right)^{2}[f(x)+f(y)] \\
& -\frac{1}{2} a(a+1)^{2} f(x+y),
\end{aligned}
$$

for $x$ and $y$ in $X$.
Theorem 12. Suppose that there exists a mapping $\phi: X^{2} \rightarrow$ $\mathbb{R}^{+}:=[0, \infty)$ for which a mapping $f: X \rightarrow Y$ satisfies $f(0)=$ 0 and

$$
\left\|D_{a} f(x, y)\right\|_{Y} \leq \phi(x, y),
$$

and the series $\sum_{j=0}^{\infty}\left(K /|a|^{4 \beta}\right)^{j} \phi\left(a^{j} x, a^{j} y\right)$ converges for all $x, y \in X$. Then there exists a unique Euler-Lagrange-Rassias quartic mapping $Q: X \rightarrow Y$ which satisfies (7) and the inequality

$$
\|f(x)-Q(x)\|_{Y} \leq \frac{K}{|a|^{4 \beta}} \sum_{j=0}^{\infty}\left(\frac{K}{|a|^{4 \beta}}\right)^{j} \phi\left(a^{j} x, 0\right),
$$

for all $x \in X$.

Proof. By letting $y=0$ in (22) and $f(0)=0$, we have

$$
\begin{gathered}
\| f(a x)+f(x)+\frac{1}{2} a(a-1)^{2} f(x)-\frac{1}{2} a(a+1)^{2} \\
-\left(a^{2}-1\right)^{2} f(x) \|_{Y} \\
=\left\|f(a x)-a^{4} f(x)\right\|_{Y} \\
=|a|^{4 \beta}\left\|f(x)-\frac{1}{a^{4}} f(a x)\right\|_{Y} \leq \phi(x, 0),
\end{gathered}
$$

that is,

$$
\left\|f(x)-\frac{1}{a^{4}} f(a x)\right\|_{Y} \leq \frac{1}{|a|^{4 \beta}} \phi(x, 0),
$$

for all $x \in X$. For any positive integer $m$, we have

$$
\begin{gathered}
\left\|\left(\frac{1}{a^{4}}\right)^{m} f\left(a^{m} x\right)-\left(\frac{1}{a^{4}}\right)^{m+1} f\left(a^{m+1} x\right)\right\|_{Y} \\
\leq \frac{1}{|a|^{4 \beta}}\left(\frac{1}{|a|^{4 \beta}}\right)^{m} \phi\left(a^{m} x, 0\right),
\end{gathered}
$$

for all $x \in X$. For any positive integers $n$ and $m$ with $m<n$,

$$
\begin{aligned}
& \left\|\left(\frac{1}{a^{4}}\right)^{m} f\left(a^{m} x\right)-\left(\frac{1}{a^{4}}\right)^{n} f\left(a^{n} x\right)\right\|_{Y} \\
& \leq \frac{1}{K^{m-1}} \frac{1}{|a|^{4 \beta}} \sum_{j=m}^{n-1}\left(\frac{K}{|a|^{4 \beta}}\right)^{j} \phi\left(a^{j} x, 0\right),
\end{aligned}
$$

for all $x \in X$. By letting $m=0$, we have

$$
\begin{aligned}
\| f(x) & -\left(\frac{1}{a^{4}}\right)^{n} f\left(a^{n} x\right) \|_{Y} \\
\leq & \frac{K}{|a|^{4 \beta}} \sum_{j=0}^{n-1}\left(\frac{K}{|a|^{4 \beta}}\right)^{j} \phi\left(a^{j} x, 0\right),
\end{aligned}
$$

for all $x \in X$ and $n \in \mathbb{N}$. Since the right-hand side of the previous inequality tends to 0 as $n \rightarrow \infty,\left\{\left(1 / a^{4}\right)^{n} f\left(a^{n} x\right)\right\}$ is 
a Cauchy sequence in the quasi $\beta$-Banach space $Y$. Thus we may define

$$
Q(x)=\lim _{n \rightarrow \infty}\left(\frac{1}{a^{4}}\right)^{n} f\left(a^{n} x\right)
$$

for all $x \in X$. Hence we have the inequality (23). Since $K \geq 1$, replacing $x$ and $y$ by $a^{n} x$ and $a^{n} y$, respectively, and dividing by $|a|^{4 \beta n}$ in (22), we have

$$
\left(\frac{1}{|a|^{4 \beta}}\right)^{n}\left\|D_{a} f\left(a^{n} x, a^{n} y\right)\right\|_{Y} \leq\left(\frac{K}{|a|^{4 \beta}}\right)^{n} \phi\left(a^{n} x, a^{n} y\right),
$$

for all $x, y \in X$. By taking $n \rightarrow \infty$, the definition of $Q$ implies that $Q$ satisfies (7) for all $x, y \in X$; that is, $Q$ is the EulerLagrange-Rassias quartic mapping. It is left to show that the quadratic mapping $Q$ is unique. Assume that there exists $T$ : $X \rightarrow Y$ satisfying (7) and (23). Then

$$
\begin{aligned}
\|T(x)-Q(x)\|_{Y} & =\left(\frac{1}{|a|^{4 \beta}}\right)^{n}\left\|T\left(a^{n} x\right)-Q\left(a^{n} x\right)\right\|_{Y} \\
& \leq\left(\frac{1}{|a|^{4 \beta}}\right)^{n} K\left(\left\|T\left(a^{n} x\right)-f\left(a^{n} x\right)\right\|_{Y}\right. \\
& \left.+\left\|f\left(a^{n} x\right)-Q\left(a^{n} x\right)\right\|_{Y}\right) \\
& \leq \frac{2}{|a|^{4 \beta n}} \sum_{j=n}^{\infty}\left(\frac{K}{|a|^{4 \beta}}\right)^{j} \phi\left(a^{j} x, 0\right),
\end{aligned}
$$

for all $x \in X$. By letting $n \rightarrow \infty$, we immediately have the uniqueness of $Q$.

Theorem 13. Suppose that there exists a mapping $\phi: X^{2} \rightarrow$ $\mathbb{R}^{+}:=[0, \infty)$ for which a mapping $f: X \rightarrow Y$ satisfies $f(0)=$ 0 and

$$
\left\|D_{a} f(x, y)\right\|_{Y} \leq \phi(x, y)
$$

and the series $\sum_{j=1}^{\infty}\left(|a|^{4 \beta} K\right)^{j} \phi\left(a^{-j} x, a^{-j} y\right)$ converges for all $x, y \in X$. Then there exists a unique Euler-Lagrange-Rassias quartic mapping $Q: X \rightarrow Y$ which satisfies (7) and the inequality

$$
\|f(x)-Q(x)\|_{Y} \leq \sum_{j=1}^{\infty}\left(|a|^{4 \beta} K\right)^{j} \phi\left(a^{-j} x, 0\right),
$$

for all $x \in X$.

Proof. If $x$ is replaced by $(1 / a) x$ in inequality (25), we have

$$
\left\|f(x)-a^{4} f\left(\frac{1}{a} x\right)\right\|_{Y} \leq \phi(x, 0),
$$

for all $x \in X$. The remains of the proof follow from the proof of Theorem 12.

\section{Stability in Quasi Fuzzy $\beta$-Normed Spaces}

Let us fix some notations which will be used throughout this section. We assume $X$ is a vector space and $(Y, N)$ is a quasi fuzzy $\beta$-Banach space. We will prove the Hyers-Ulam-Rassias stability of the functional equation satisfying equation (7) in quasi fuzzy $\beta$-Banach space.

Theorem 14. Let $\phi: X^{2} \rightarrow[0, \infty)$ be a function such that for some $0<|\alpha|<|a|^{4}$

$$
\begin{aligned}
& N^{\prime}(\phi(a x, 0), t) \geq N^{\prime}(\alpha \phi(x, 0), t), \\
& \lim _{n \rightarrow \infty} N^{\prime}\left(\phi\left(a^{n} x, a^{n} y\right),|a|^{4 n \beta} t\right)=1,
\end{aligned}
$$

for all $x, y \in X$ and all $t>0$. Let $f: X \rightarrow Y$ be a mapping satisfying $f(0)=0$ and

$$
N\left(D_{a} f(x, y), t\right) \geq N^{\prime}(\phi(x, y), t),
$$

for all $x, y \in X$ and all $t>0$.

Then $Q(x):=N-\lim _{n \rightarrow \infty}\left(1 / a^{4 n}\right) f\left(a^{n} x\right)$ exists for each $x \in X$ and defines a unique Euler-Lagrange-Rassias quartic mapping $Q: X \rightarrow Y$ such that

$$
N(f(x)-Q(x), t) \geq N^{\prime}\left(\phi(x, 0), \frac{|a|^{4 \beta}-|\alpha|^{\beta}}{2} t\right),
$$

for all $x \in X$ and all $t>0$.

Proof. Let $y=0$ in inequality (36). Since $f(0)=0$, we have

$$
\begin{aligned}
& N\left(D_{a} f(x, 0), t\right) \\
& \quad=N\left(\frac{1}{a^{4}} f(a x)-f(x), \frac{t}{|a|^{4 \beta}}\right) \geq N^{\prime}(\phi(x, 0), t),
\end{aligned}
$$

for all $x \in X$ and all $t>0$. Replacing $x$ by $a^{n} x$ in inequality (38),

$$
N\left(\frac{1}{a^{4}} f\left(a^{n+1} x\right)-f\left(a^{n} x\right), \frac{t}{|a|^{4 \beta}}\right) \geq N^{\prime}\left(\phi\left(a^{n} x, 0\right), t\right),
$$

that is,

$$
\begin{aligned}
& N\left(\left(\frac{1}{a^{4}}\right)^{n+1} f\left(a^{n+1} x\right)-\left(\frac{1}{a^{4}}\right)^{n} f\left(a^{n} x\right), \frac{t}{|a|^{4 \beta}} \frac{1}{|a|^{4 n \beta}}\right) \\
& \geq N^{\prime}\left(\phi\left(a^{n} x, 0\right), t\right),
\end{aligned}
$$

for all $x \in X, t>0$ and $n \geq 0$. Since $N^{\prime}\left(\phi\left(a^{n} x, 0\right), t\right) \geq$ $N^{\prime}\left(\phi(x, 0),\left(t /|\alpha|^{n \beta}\right)\right)$,

$$
\begin{aligned}
& N\left(\left(\frac{1}{a^{4}}\right)^{n+1} f\left(a^{n+1} x\right)-\left(\frac{1}{a^{4}}\right)^{n} f\left(a^{n} x\right), \frac{t}{|a|^{4 \beta}} \frac{1}{|a|^{4 n \beta}}\right) \\
& \geq N^{\prime}\left(\phi(x, 0), \frac{t}{|\alpha|^{n \beta}}\right) .
\end{aligned}
$$


By letting $t=|\alpha|^{n \beta} t$ in the previous inequality,

$$
\begin{aligned}
& N\left(\left(\frac{1}{a^{4}}\right)^{n+1} f\left(a^{n+1} x\right)-\left(\frac{1}{a^{4}}\right)^{n} f\left(a^{n} x\right), \frac{t}{|a|^{4 \beta}} \cdot\left(\frac{|\alpha|}{|a|^{4}}\right)^{n \beta}\right) \\
& \geq N^{\prime}(\phi(x, 0), t),
\end{aligned}
$$

for all $x \in X$ and all $t>0$. Hence we get

$$
\begin{aligned}
& N\left(\left(\frac{1}{a^{4}}\right)^{n} f\left(a^{n} x\right)-f(x), \sum_{j=0}^{n-1} \frac{t}{|a|^{4 \beta}} \cdot\left(\frac{|\alpha|}{|a|^{4}}\right)^{j \beta}\right) \\
& \geq \min \bigcup_{j=0}^{n-1}\left[N \left(\left(\frac{1}{a^{4}}\right)^{j+1} f\left(a^{j+1} x\right)\right.\right. \\
& \left.\left.\quad-\left(\frac{1}{a^{4}}\right)^{j} f\left(a^{j} x\right), \frac{t}{|a|^{4 \beta}} \cdot\left(\frac{|\alpha|}{|a|^{4}}\right)^{j \beta}\right)\right] \\
& \geq N^{\prime}(\phi(x, 0), t),
\end{aligned}
$$

for all $x \in X$ and all $t>0$. Letting $x=a^{m} x$ in the previous inequality, we have

$$
\begin{gathered}
N\left(\left(\frac{1}{a^{4}}\right)^{n} f\left(a^{n+m} x\right)-f\left(a^{m} x\right), \sum_{j=0}^{n-1} \frac{t}{|a|^{4 \beta}} \cdot\left(\frac{|\alpha|}{|a|^{4}}\right)^{j \beta}\right) \\
\geq N^{\prime}\left(\phi\left(a^{m} x, 0\right), t\right) \geq N^{\prime}\left(\phi(x, 0), \frac{t}{|\alpha|^{m \beta}}\right),
\end{gathered}
$$

that is,

$$
\begin{gathered}
N\left(\left(\frac{1}{a^{4}}\right)^{n+m} f\left(a^{n+m} x\right)-\left(\frac{1}{a^{4}}\right)^{m} f\left(a^{m} x\right),\right. \\
\left.\frac{t}{|a|^{4 m \beta}} \sum_{j=0}^{n-1} \frac{1}{|a|^{4 \beta}} \cdot\left(\frac{|\alpha|}{|a|^{4}}\right)^{j \beta}\right) \\
\geq N^{\prime}\left(\phi(x, 0), \frac{t}{|\alpha|^{m \beta}}\right),
\end{gathered}
$$

for all $x \in X$ and all $t>0$. Letting $t=|\alpha|^{m \beta} t$, we have

$$
\begin{gathered}
N\left(\left(\frac{1}{a^{4}}\right)^{n+m} f\left(a^{n+m} x\right)-\left(\frac{1}{a^{4}}\right)^{m} f\left(a^{m} x\right),\right. \\
\left.\frac{t}{|a|^{4 \beta}} \sum_{j=m}^{n+m-1}\left(\frac{|\alpha|}{|a|^{4}}\right)^{j \beta}\right) \\
\geq N^{\prime}(\phi(x, 0), t),
\end{gathered}
$$

for all $x \in X, t>0$ and $n, m \geq 0$. Hence $\left\{\left(1 / a^{4 n}\right) f\left(a^{n} x\right)\right\}$ is a Cauchy sequence in the quasi fuzzy $\beta$-Banach space $(Y, N)$. Thus, we may define

$$
Q(x)=N-\lim _{n \rightarrow \infty} \frac{1}{a^{4 n}} f\left(a^{n} x\right)
$$

for all $x \in X$. Hence inequality (43) implies that

$$
\begin{gathered}
N(Q(x)-f(x), t) \\
\geq \min \left\{N\left(Q(x)-\left(\frac{1}{a^{4}}\right)^{n} f\left(a^{n} x\right), \frac{t}{2}\right),\right. \\
\left.N\left(\left(\frac{1}{a^{4}}\right)^{n} f\left(a^{n} x\right)-f(x), \frac{t}{2}\right)\right\} \\
\geq N^{\prime}\left(\phi(x, 0), \frac{t}{\left(2 /|a|^{4 \beta}\right) \sum_{j=0}^{n-1}\left(|\alpha| /|a|^{4}\right)^{j \beta}}\right),
\end{gathered}
$$

for $n$ large enough and all $x \in X$. Taking the limit as $n \rightarrow \infty$ and using $\left(N_{6}\right)$, we have

$$
N(Q(x)-f(x), t) \geq N^{\prime}\left(\phi(x, 0), \frac{|a|^{4 \beta}-|\alpha|^{\beta}}{2} t\right),
$$

for all $x \in X$. Hence it satisfies inequality (37). Now letting $x=a^{n} x$ and $y=a^{n} y$ in (36),

$$
N\left(D_{a} f\left(a^{n} x, a^{n} y\right), t\right) \geq N^{\prime}\left(\phi\left(a^{n} n, a^{n} y\right), t\right),
$$

for all $x \in X$ and all $t>0$. This implies that

$$
N\left(\left(\frac{1}{a^{4}}\right)^{n} D_{a} f\left(a^{n} x, a^{n} y\right), t\right) \geq N^{\prime}\left(\phi\left(a^{n} n, a^{n} y\right),|a|^{4 \beta} t\right),
$$

for all $x \in X$ and all $t>0$. Since $N^{\prime}\left(\phi\left(a^{n} n, a^{n} y\right),|a|^{4 \beta} t\right)=1$, we may conclude that the mapping $Q$ satisfies (7); that is, $Q$ is the Euler-Lagrange-Rassias quartic mapping. It is left to show that the quartic mapping $Q$ is unique. Assume there is another $T: X \rightarrow Y$ satisfying (7) and inequality (37). For each $x \in X$, clearly $Q\left(a^{n} x\right)=a^{4 n} Q(x)$ and $T\left(a^{n} x\right)=a^{4 n} T(x)$ for all $n \in \mathbb{N}$.

$$
\begin{aligned}
N(T(x)-Q(x), t) & =N\left(\frac{1}{a^{4 n}} T\left(a^{n} x\right)-\frac{1}{a^{4 n}} Q\left(a^{n} x\right), t\right) \\
& =N\left(T\left(a^{n} x\right)-Q\left(a^{n} x\right),|a|^{4 n \beta} t\right)
\end{aligned}
$$




$$
\begin{aligned}
& \geq \min \left\{N\left(T\left(a^{n} x\right)-f\left(a^{n} x\right), \frac{|a|^{4 n \beta} t}{2}\right),\right. \\
& N\left(f\left(a^{n} x\right)-Q\left(a^{n} x\right),\right. \\
& \left.\left.\frac{|a|^{4 n \beta} t}{2}\right)\right\} \\
& \geq N^{\prime}\left(\phi\left(a^{n} x, 0\right), \frac{|a|^{4 \beta}-|\alpha|^{\beta}}{2} \cdot \frac{|a|^{4 n \beta} t}{2}\right) \\
& \geq N^{\prime}\left(\phi(x, 0), \frac{|a|^{4 \beta}-|\alpha|^{\beta}}{4} t\right), \\
& \left.\left(\frac{|a|^{4}}{|\alpha|}\right)^{n \beta} \cdot \frac{\mid{ }^{4 \beta}}{2}\right)
\end{aligned}
$$

for all $x \in X$ and $t>0$. Since $0<|\alpha|<|a|^{4}$, we have $\lim _{n \rightarrow \infty}\left(|a|^{4} /|\alpha|\right)^{n \beta}=\infty$. Hence $N(T(x)-Q(x), t)=1$; that is, the mapping $Q$ is unique, as desired.

Theorem 15. Let $\phi: X^{2} \rightarrow[0, \infty)$ be a function such that for some $|\alpha|>|a|^{4}$

$$
\begin{aligned}
& N^{\prime}\left(\phi\left(\frac{1}{a} x, 0\right), t\right) \geq N^{\prime}(\alpha \phi(x, 0), t), \\
& \lim _{n \rightarrow \infty} N^{\prime}\left(\phi\left(a^{-n} x, a^{-n} y\right), \frac{1}{|a|^{4 n \beta}} t\right)=1,
\end{aligned}
$$

for all $x, y \in X$ and all $t>0$. Let $f: X \rightarrow Y$ be a mapping satisfying $f(0)=0$ and

$$
N\left(D_{a} f(x, y), t\right) \geq N^{\prime}(\phi(x, y), t),
$$

for all $x, y \in X$ and all $t>0$.

Then $Q(x):=N-\lim _{n \rightarrow \infty} a^{4 n} f\left(\left(1 / a^{n}\right) x\right)$ exists for each $x \in X$ and defines a unique Euler-Lagrange-Rassias quartic mapping $Q: X \rightarrow Y$ such that

$$
N(f(x)-Q(x), t) \geq N^{\prime}\left(\phi(x, 0), \frac{|\alpha|^{\beta}-|a|^{4 \beta}}{2|\alpha|^{2 \beta}} t\right),
$$

for all $x \in X$ and all $t>0$.

Proof. The techniques are completely similar to the proof of Theorem 14. Hence we present some key idea of this proof. Let $y=0$ in inequality (54). Since $f(0)=0$, we have

$$
\begin{aligned}
& N\left(D_{a} f(x, 0), t\right) \\
& \quad=N\left(f(a x)-a^{4} f(x), t\right) \geq N^{\prime}(\phi(x, 0), t),
\end{aligned}
$$

for all $x \in X$ and all $t>0$. Replacing $x$ by $(1 / a) x$ in inequality (56), we have

$$
\begin{aligned}
& N\left(f(x)-a^{4} f\left(\frac{1}{a} x\right), t\right) \\
& \quad \geq N^{\prime}\left(\phi\left(\frac{1}{a} x, 0\right), t\right) \geq N^{\prime}\left(\phi(x, 0), \frac{1}{|\alpha|^{\beta}} t\right)
\end{aligned}
$$

or

$$
N\left(f(x)-a^{4} f\left(\frac{1}{a} x\right),|\alpha|^{\beta} t\right) \geq N^{\prime}(\phi(x, 0), t),
$$

for all $x \in X$ and all $t>0$. For positive integers $n$ and $m$,

$$
\begin{aligned}
& N\left(a^{4(n+m)} f\left(\frac{1}{a^{n+m}} x\right)-a^{4 m} f\left(\frac{1}{a^{m}} x\right),\right. \\
& \left.|\alpha|^{\beta} \sum_{j=m}^{n+m-1}\left(\frac{|a|^{4}}{|\alpha|}\right)^{j \beta} t\right) \geq N^{\prime}(\phi(x, 0), t),
\end{aligned}
$$

for all $x \in X$ and $t>0$. Hence we may conclude that $\left\{a^{4 n} f\left(\left(1 / a^{n}\right) x\right)\right\}$ is a Cauchy sequence in the quasi fuzzy $\beta$ Banach space $(Y, N)$. Thus we may define

$$
Q(x)=N-\lim _{n \rightarrow \infty} a^{4 n} f\left(\frac{1}{a^{n}} x\right),
$$

for all $x \in X$. Also, for any positive integer $n$, we get

$$
\begin{aligned}
& N\left(a^{4 n} f\left(\frac{1}{a^{n}} x\right)-f(x), t\right) \\
& \quad \geq N^{\prime}\left(\phi(x, 0), \frac{t}{|\alpha|^{\beta} \sum_{j=0}^{n-1}\left(|a|^{4} /|\alpha|\right)^{j \beta}}\right),
\end{aligned}
$$

for all $x \in X$ and all $t>0$. This implies inequality (55).

\section{Acknowledgment}

The present research was conducted by the research fund of Dankook University in 2013.

\section{References}

[1] S. M. Ulam, A Collection of Mathematical Problems, Interscience Publishers, New York, NY, USA, 1960.

[2] D. H. Hyers, "On the stability of the linear functional equation," Proceedings of the National Academy of Sciences of the United States of America, vol. 27, pp. 222-224, 1941.

[3] T. Aoki, "On the stability of the linear transformation in Banach spaces," Journal of the Mathematical Society of Japan, vol. 2, pp. 64-66, 1950.

[4] T. M. Rassias, "On the stability of the linear mapping in Banach spaces," Proceedings of the American Mathematical Society, vol. 72, no. 2, pp. 297-300, 1978.

[5] Z. Gajda, "On stability of additive mappings," International Journal of Mathematics and Mathematical Sciences, vol. 14, no. 3, pp. 431-434, 1991.

[6] S. Czerwik, "On the stability of the quadratic mapping in normed spaces," Abhandlungen aus dem Mathematischen Seminar der Universität Hamburg, vol. 62, pp. 59-64, 1992.

[7] T. M. Rassias, "On the stability of functional equations in Banach spaces," Journal of Mathematical Analysis and Applications, vol. 251, no. 1, pp. 264-284, 2000.

[8] T. M. Rassias and P. Šemrl, "On the Hyers-Ulam stability of linear mappings," Journal of Mathematical Analysis and Applications, vol. 173, no. 2, pp. 325-338, 1993. 
[9] T. M. Rassias and K. Shibata, "Variational problem of some quadratic functionals in complex analysis," Journal of Mathematical Analysis and Applications, vol. 228, no. 1, pp. 234-253, 1998.

[10] J.-H. Bae and W.-G. Park, "On the generalized Hyers-UlamRassias stability in Banach modules over a $C^{x}$-algebra," Journal of Mathematical Analysis and Applications, vol. 294, no. 1, pp. 196-205, 2004.

[11] J. M. Rassias, "On the stability of the Euler-Lagrange functional equation," Chinese Journal of Mathematics, vol. 20, no. 2, pp. 185-190, 1992.

[12] K.-W. Jun and H.-M. Kim, "On the stability of Euler-Lagrange type cubic mappings in quasi-Banach spaces," Journal of Mathematical Analysis and Applications, vol. 332, no. 2, pp. 1335-1350, 2007.

[13] H.-M. Kim and M.-Y. Kim, "Generalized stability of EulerLagrange quadratic functional equation," Abstract and Applied Analysis, vol. 2012, Article ID 219435, 16 pages, 2012.

[14] A. Zivari-Kazempour and M. E. Gordji, "Generalized HyersUlam stabilities of an Euler-Lagrange-Rassias quadratic functional equation," Asian-European Journal of Mathematics, vol. 5, no. 1, Article ID 1250014, 7 pages, 2012.

[15] T. Z. Xu and J. M. Rassias, "Stability of general multi-EulerLagrange quadratic functional equations in non-archimedean fuzzy normed spaces," Advances in Difference Equations, vol. 2012, no. 1, 2012.

[16] J. M. Rassias, "Solution of the Ulam stability problem for quartic mappings," Glasnik Matematički, vol. 34, no. 2, pp. 243-252, 1999.

[17] J. K. Chung and P. K. Sahoo, "On the general solution of a quartic functional equation," Bulletin of the Korean Mathematical Society, vol. 40, no. 4, pp. 565-576, 2003.

[18] Y.-S. Lee and S.-Y. Chung, "Stability of quartic functional equations in the spaces of generalized functions," Advances in Difference Equations, vol. 2009, Article ID 838347, 16 pages, 2009.

[19] Y. Benyamini and J. Lindenstrauss, Geometric Nonlinear Functional Analysis. Vol. 1, vol. 48, American Mathematical Society, Providence, RI, USA, 2000.

[20] S. Rolewicz, Metric Linear Spaces, Polish Scientific Publishers, Warsaw, Poland, 2nd edition, 1984.

[21] A. K. Katsaras, "Fuzzy topological vector spaces. II," Fuzzy Sets and Systems, vol. 12, no. 2, pp. 143-154, 1984.

[22] C. Wu and J. Fang, "Fuzzy generalization of Klomogoros theorem," Journal of Harbin Institute of Technology, vol. 1, pp. $1-7,1984$.

[23] T. Bag and S. K. Samanta, "Finite dimensional fuzzy normed linear spaces," Journal of Fuzzy Mathematics, vol. 11, no. 3, pp. 687-705, 2003.

[24] C. Felbin, "Finite-dimensional fuzzy normed linear space," Fuzzy Sets and Systems, vol. 48, no. 2, pp. 239-248, 1992.

[25] S. V. Krishna and K. K. M. Sarma, "Separation of fuzzy normed linear spaces,” Fuzzy Sets and Systems, vol. 63, no. 2, pp. 207-217, 1994.

[26] J.-Z. Xiao and X.-H. Zhu, "Fuzzy normed space of operators and its completeness," Fuzzy Sets and Systems, vol. 133, no. 3, pp. 389-399, 2003.

[27] A. K. Mirmostafaee, M. Mirzavaziri, and M. S. Moslehian, "Fuzzy stability of the Jensen functional equation," Fuzzy Sets and Systems, vol. 159, no. 6, pp. 730-738, 2008.
[28] S. C. Cheng and J. N. Mordeson, "Fuzzy linear operators and fuzzy normed linear spaces," Bulletin of the Calcutta Mathematical Society, vol. 86, no. 5, pp. 429-436, 1994.

[29] A. K. Mirmostafaee and M. S. Moslehian, "Fuzzy versions of Hyers-Ulam-Rassias theorem," Fuzzy Sets and Systems, vol. 159, no. 6, pp. 720-729, 2008.

[30] A. K. Mirmostafaee, "A fixed point approach to almost quartic mappings in quasi fuzzy normed spaces," Fuzzy Sets and Systems, vol. 160, no. 11, pp. 1653-1662, 2009.

[31] H.-M. Kim, "On the stability problem for a mixed type of quartic and quadratic functional equation," Journal of Mathematical Analysis and Applications, vol. 324, no. 1, pp. 358-372, 2006.

[32] K. Jun and H. Kim, "Solution of Ulam stability problem for approximately biquadratic mappings and functional inequalities," Journal of Inequalities and Applications. In press. 


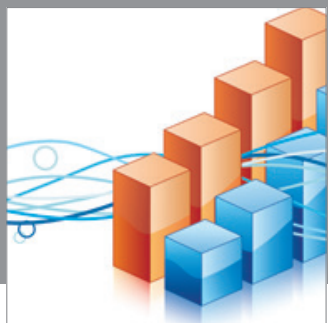

Advances in

Operations Research

mansans

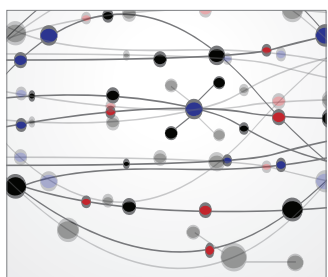

The Scientific World Journal
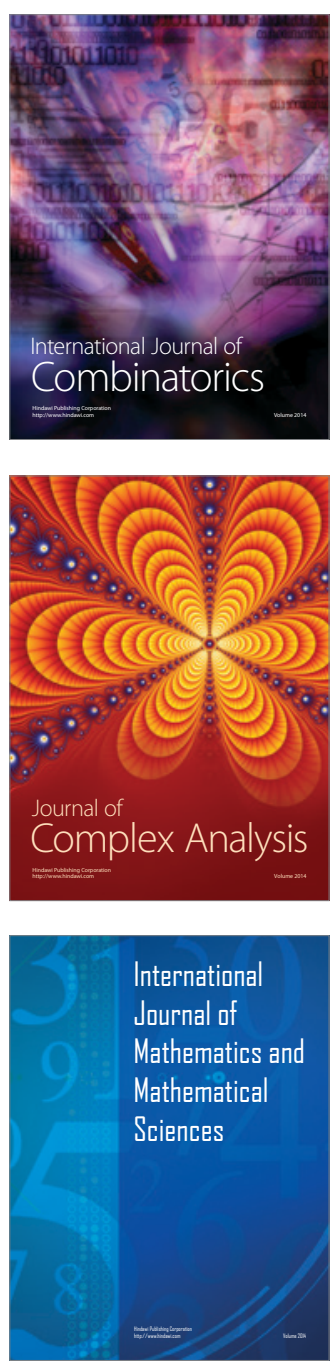
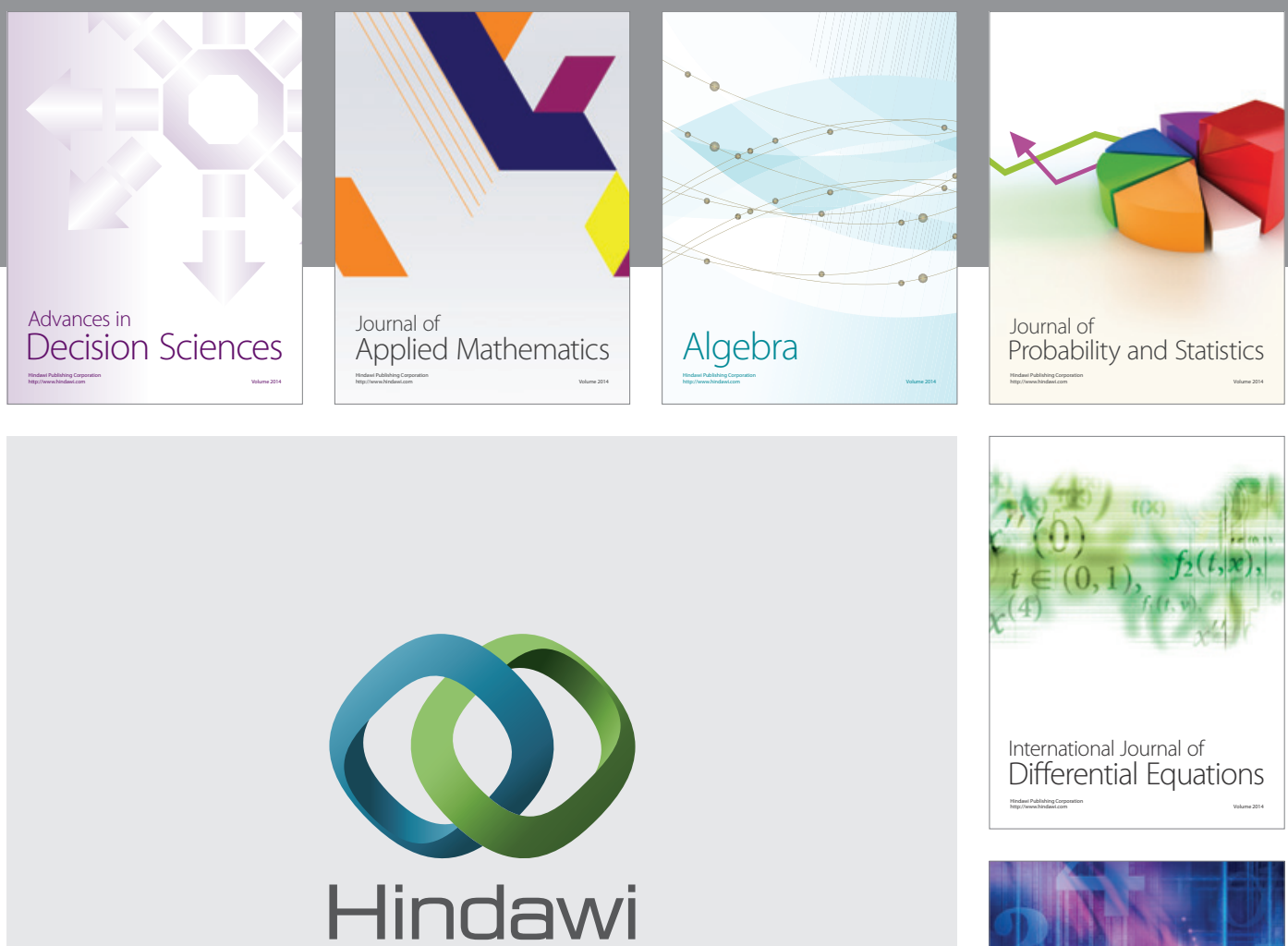

Submit your manuscripts at http://www.hindawi.com
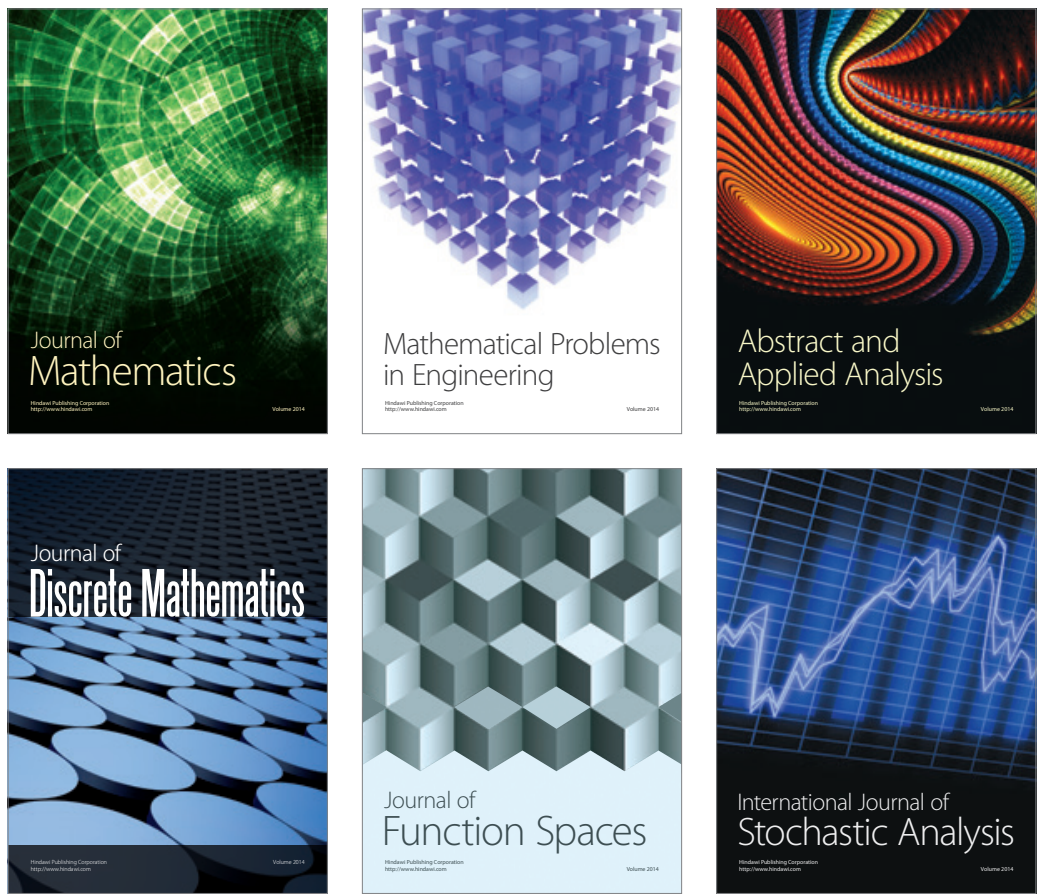

Journal of

Function Spaces

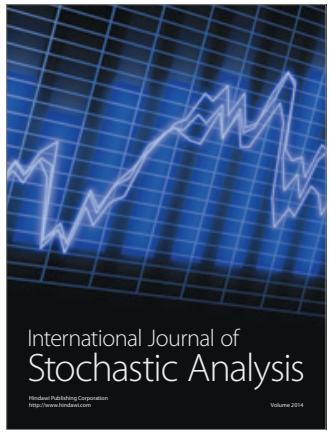

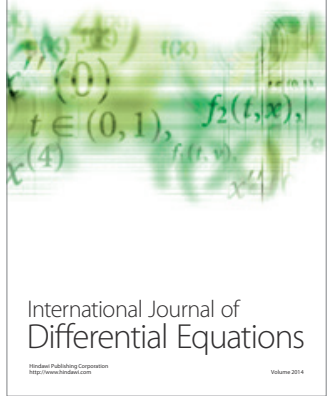
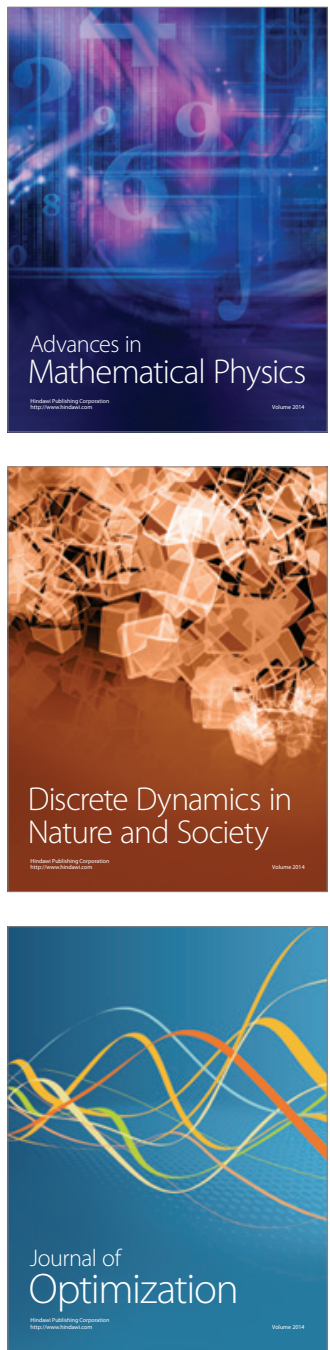